\title{
1 Sugar Signaling Induces Dynamic Changes during Meristem Development in
}

\section{Arabidopsis}

3 Magdalena Musialak-Lange ${ }^{1}$, Katharina Fiddeke ${ }^{1, \dagger, \#}$, Annika Franke $^{1, \dagger, \#}$, Friedrich Kragler $^{1}$,

\section{Affiliations:}

$6 \quad{ }^{1}$ Max Planck Institute of Molecular Plant Physiology, Department of Metabolic Networks, Am

7 Mühlenberg 1, Potsdam, 14476, Germany.

$8{ }^{\dagger}$ Current work addresses: ProBioGen AG, Herbert-Bayer-Str. 8, 13086 Berlin (K.F.) and Takeda

$9 \mathrm{GmbH}$, Lehnizstra. 70-98, 16515 Oranienburg (A.F.)

$10 \quad{ }^{\#}$ K.F. and A.F. contributed equally

$11 *$ Correspondence to: vanessa.wahl@mpimp-golm.mpg.de.

\section{One Sentence Summary:}

13 The increase in meristem size during the floral transition is regulated via a feedback regulation

14 involving sugar signaling. 
16 (SAM). This population of stem cells is maintained via a negative feedback loop involving stable expression of WUSCHEL (WUS) and CLAVATA3. SAM size is dynamic and undergoes a more than

18 2-fold expansion upon the transition to reproductive growth. The underlying mechanism

19 controlling this process is largely unknown, but coinciding increased levels of trehalose 6-

20 phosphate (T6P) suggest a participation of sugar signaling. Here we show that TREHALOSE

21 PHOSPHATE PHOSPHATASE J, a component of the T6P pathway, is directly regulated by WUS,

22 and controls SAM expansion at floral transition through WUS. Our findings demonstrate a

23 dynamic feedback-regulation between central meristem maintenance and flowering time

24 regulators with sugar signaling. 
26 in $\operatorname{size}^{1}$ (Fig. 1a). Since the transition between developmental stages requires a massive

27 reorganization of organ development and sufficient energy, it is tightly controlled by

28 environmental conditions and availability of nutrients ${ }^{2-5}$. Hence, plant development as a whole

29 and SAM maintenance in particular demands continuous cross talk between its regulatory

30 processes and the available resources. The sugar phosphate, trehalose 6-phosphate (T6P), serves

31 as a signal for sucrose availability, which is conveyed to downstream metabolic and growth

32 responses through a still largely unknown mechanism ${ }^{6}$. Also knowledge on the underlying

33 mechanism controlling the morphological processes at the SAM at floral transition is scarce.

34 However, increased levels of T6P coincide with the floral transition and the T6P pathway

35 induces flowering by regulating key flowering genes expressed in leaves and the $\mathrm{SAM}^{4}$, which

36 suggests a participation of sugar signaling in regulating the SAM size changes throughout the

37 floral transition.

To assess whether the morphological changes at the SAM involve the T6P pathway, we

investigated the effect on SAM architecture by decreasing TPS1, coding for the T6P synthesizing

S1). We compared these lines with plants with increased TPS1 in the central zone (CLV3:TPS1,

42 Fig. S2). These have smaller and bigger vegetative and reproductive meristems (Fig. 1b, 1c),

43 resulting in smaller and bigger plants, respectively ${ }^{4}$. The homeodomain transcription factor WUS

44 and the signaling peptide CLV3 non-cell autonomously contribute to communication of position-

45 dependent properties among SAM cells in a negative feedback loop and maintain the SAM cell

46 population ${ }^{7}$. We therefore analyzed their expression in the SAM with altered TPS1. While the

47 expression of WUS in the organizing centre (OC) and $C L V 3$ in the central zone (CZ) harboring 
48 the stem cells are considered mutually exclusive ${ }^{7}$, we found reduced TPS1 levels

49 (35S:amiRTPS1) lead to an enlarged OC domain marked by WUS (Fig. 1d) that overlaps with the

$50 \mathrm{CZ}$, marked by $C L V 3$ expression (Fig. 1D). Plants overexpressing TPS1 (35S:TPS1, Fig. S2)

51 display an increased stem cell pool with otherwise little effect on $W U S$ expression (Fig. 1d).

52 However, the size of the CZ decreases in plants expressing TPS1 under the CLV3 promoter (Fig.

53 1d, Fig. S2), indicating a smaller stem cell pool in support of a much smaller SAM size

54 phenotype (Fig. 1d) of the very early flowering CLV3:TPS1 plants ${ }^{4}$. The expression of

55 HISTONE4, CYCLIN D3;1 and CYCLIN-DEPENDENT KINASE2;1, three cell cycle marker

56 genes (Fig. S3), suggests a higher proliferation rate and an increased number of cells displaced

57 from the $\mathrm{CZ}$ to adopt organ-specific cell fates at the periphery as a plausible reason for the

58 decreased SAM size of CLV3:TPS1 and vice versa increased SAM size of 35S:amiRTPS1 plants

59 (Fig. 1b, 1c).

To assess whether the WUS/CLV feedback loop can uncouple in a wild-type SAM

61 during normal growth, we analyzed $W U S$ and $C L V 3$ expression in wild-type plants in a time

62 series spanning the floral transition (Fig. 1e). Throughout the vegetative phase SAM size

63 gradually increases due to rising cell numbers (Fig. 1a; Fig. S4) ${ }^{8}$, reaching its maximum at the

64 floral transition, independent of whether the plants are grown in continuous long days (LD) (Fig.

65 1a) or in short days (SD) followed by a transfer to LD (Fig. S5). An enlarging SAM at the floral

66 transition correlates with a larger WUS expression domain expanding into the $\mathrm{CZ}$ and the outer

67 cell layer (L1) (Fig. 1e-g). This is supported by larger meristems of the 35S:amiRTPS1 line,

68 which express WUS in the central zone (Fig. 1d). This observation is also consistent with

69 massive proliferation of meristematic cells detected when $W U S$ is expressed from a CLV3

70 promoter in the $\mathrm{CZ}$ that resulted in meristems, no longer producing organs ${ }^{9}$. The presence of 
71 WUS transcript in the outer meristem layers might be explained by a transient cytokinin signal in

$72 \mathrm{~L} 1$, which induces $W U S$ and was reported to respond to carbon in seedlings ${ }^{10-12}$. However,

73 cytokinin levels seem not to be altered in the L1 layer of cells as indicated by the synthetic 74 cytokinin reporter TCSn:GFP ${ }^{13}$ (Fig. S6). Further, while WUS expands to L1 for a short period

75 (8-10 DAG), the CLV3 expression domain remains expanded post floral transition (Fig. 1e, 1h).

76 This suggests a transient uncoupling, which is re-established at the reproductive SAM (16 DAG),

77 resembling earlier vegetative SAM expression patterns (6 DAG) (Fig. 1e), allowing organ 78 production to resume.

To understand how the T6P pathway might control this process, we first analyzed expression of the ten genes encoding TREHALOSE PHOSPHATE PHOSPHATASEs (TPP) ${ }^{6}$.

81 Except for $T P P C$ and $T P P D$, which are expressed below detection limit, all were expressed in 82 distinct SAM domains (Fig. S7). TPPJ strongly increases in the enlarged SAM of clv3-7 due to 83 an ectopic expression in L1 and L2 (Fig. 2a-c, Fig. S8, S9). Notably, in clv3-7 and clv3-10

84 mutants high levels of WUS in the outer SAM layers (Fig S9) ${ }^{14}$ coincides with ectopic expression 85 of TPPJ (Fig. S9). To assess whether ectopic expression of TPPJ contributes to the enlarged 86 SAM of the clv3 mutant, we next overexpressed TPPJ (35S:TPPJ, Fig. 2d). This results in 87 significantly enlarged SAMs (Fig. 2e), and importantly, expands the WUS expression domain 88 into L1 (Fig. 2f), consistent with our finding in wild-type plants at the floral transition (Fig. 1e).

90 SAM implies a direct influence of WUS on TPPJ expression. This is supported by an in silico 91 analysis which predicts multiple, canonical WUS binding sites in the sequence upstream of the $92 T P P J$ coding sequence $\left(T P P J^{\mathrm{WUS}}\right.$, Fig. 3a) ${ }^{10,15-17}$. WUS is a bifunctional transcription factor, 93 which was found to both activate and repress gene expression ${ }^{7}$. To understand if WUS directly 
94 controls TPPJ, we performed in vivo transactivation assays. These show that WUS activates

95 reporter gene expression when using a 2865 bp TPPJ 5' sequence, containing 17 putative $96 T P P J^{\mathrm{WUS}}$ sites (Fig. 3a). Progressive deletion of the TPPJ 5' sequence results in a reduction of 97 reporter gene activation, suggesting an additive effect of the individual TPPJ ${ }^{\mathrm{WUS}}$ sites (\#1-6; Fig.

$983 \mathrm{a}, 3 \mathrm{~b})$. We confirmed the direct binding of WUS to three distinct regions of the TPPJ promoter 99 (I, II, and III, Fig. 3c-e, Fig. S10) by Chromatin immunoprecipitation (ChIP) coupled to PCR using a specific antibody against WUS (Fig. S11), while all other regions did not indicate

101 binding (Fig. S10, Fig. S12). We observed enrichment of WUS binding to TPPJ ${ }^{\text {WUS }}$ elements up 102 to $0.67 \%$ of the input DNA in $\operatorname{clv} 3-7$ and up to $0.75 \%$ of the input DNA in $\operatorname{clv} 3-10$ apices, both 103 of which express WUS at high levels in comparison to input DNA from wild-type apices, where 104 WUS is expressed in only a few cells and input DNA from leaves, with no WUS (Fig. 3c-e; Fig. 105 S10). Lastly, electrophoretic mobility shift assays (EMSA) confirm in vitro binding of WUS to 106 the investigated sequences (I, II 1-3, III 1-3; Fig. 3f). In summary, we demonstrate that seven out 107 of 17 putative TPPJ ${ }^{\mathrm{WUS}}$ sites are directly targeted by WUS in vivo.

To further assess the role of TPPJ at the SAM we used an artificial miRNA (amiRTPPJ) approach to downregulate TPPJ (35S:amiRTPPJ) (Fig. S13). Similar to a tppj T-DNA insertion mutant, plants overexpressing either of two versions of an amiRTPPJ (V1, V2) flower 111 significantly earlier in both LD and SD (35S:amiRTPPJ, Fig. S14, Table S1). ML1 expression 112 specifically and stably localizes to the epidermis (L1) throughout the investigated developmental 113 stages (Fig. S15). ML1:amiRTPPJ plants reduce TPPJ expression in L1 (arrowheads in Fig. 114 S16), have smaller SAMs and flower significantly earlier in LD and SD (Fig. 4a-c, Fig. S17, $115 \mathrm{~S} 18$, Table S1), while the level of TPPJ reduction is proportional to the acceleration of flowering 116 (Fig. 4b and c, Fig. S13, Table S1). To date there are no reports that mutants in meristem 
117

118

119

120

121

122

123

124

125

126

127

128

129

130

131

132

maintenance genes are affected in their flowering time. We found that plants mutant for $C L V 3$ are late flowering (Fig. 4c, Table S1). However, when ML1:amiRTPPJ is introgressed into either the clv3-7 or clv3-10 background, the late flowering phenotype of the mutant plants is restored to wild type (Fig. 4c, Fig. S18, Table S1), suggesting that TPPJ expression in the epidermis is also necessary for the late flowering phenotype of clv3. Hence, the early flowering of ML1:amiRTPPJ in a wild-type background is due to a reduction of TPPJ expression in L1 in its endogenous expression domain. In addition, other prominent morphological defects of clv3-10 such as fasciated stems and enormous inflorescence SAMs are visibly reduced in the presence of ML1:amiRTPPJ (Fig. S18). CLV3 expression increases to much higher levels in ML1:amiRTPPJ than in plants overexpressing TPPJ (Fig. 4d), suggesting an active role of the T6P pathway in the outer SAM layer with regard to stem cell maintenance. In addition, $W U S$ is induced (Fig. 4d, Fig. S19), indicating uncoupling of the WUS/CLV feedback loop, consistent with our findings with altered T6P signaling at the SAM (Fig. 1d, Fig. 2f).

Since the T6P pathway influences the age pathway at the SAM ${ }^{4}$, we analyzed mature miR156 as well as the expression of SPL3, SPL4, SPL5, SPL9 and SPL15, all associated with floral transition in general ${ }^{18,19}$ (Fig. S20, Table S1). We found a strong reduction of mature miR156 levels correlating with lower TPPJ expression in ML1:amiRTPPJ, and corresponding increased expression of SPL3, SPL4, SPL5, and SPL9 (Fig. 4e). In line, miR156 abundance was highly increased in TPPJ overexpressing plants, while the corresponding SPLs were downregulated (Fig. 4e). However, expression of SPL15, previously described to be mainly responsible for integrating the aging stimulus into the regulation of the floral onset ${ }^{20}$, was not differentially expressed in either of the transgenic lines (Fig. S21). In addition, we found miR156 levels significantly increased in clv3-10 mutant apices (Fig. 4f). In response, the levels of SPL3, 
140 SPL5, and SPL9 were reduced in line with the late flowering phenotype of the mutant. In contrast

141 to what would be expected, we found more SPL4 in apices of the late flowering clv3-10 (Fig. 4f).

142 Higher order lines from spl3, spl4 and spl5 deletion mutant plants are significantly later

143 flowering in all lines with spl4 (Fig. S20, Table S1). This argues for an important role of SPL4 in

144 inducing flowering. SPL4, similar to SPL3, SPL5 and SPL9, is induced at the wild-type SAM at

145 floral transition ${ }^{21}$. However, it is expressed in the very center of the SAM, in a domain largely

146 overlapping with the WUS protein domain ${ }^{5,17,20,22}$, while $S P L 3, S P L 5$, and SPL9 are expressed at

147 the periphery of the SAM and in the vasculature of young leaves ${ }^{5,20}$. This might denote a direct

148 regulation of SPL4 by WUS, which would explain increased SPL4 in the clv3 mutant

149 background (Fig. 4f) and the previously identified partially miR156-dependent regulation of the

150 SPLs by the T6P pathway ${ }^{4}$. Indeed, we identified a larger number of potential SPL4 ${ }^{\mathrm{WUS}}$ sites

151 upstream of the SPL4 coding sequence when compared to the 5' intergenic regions of the other

152 SPLs (Fig. S22). However, this finding also suggests that additional players downstream of

153 CLV3 are important for the onset of flowering, which cannot be bypassed by an otherwise

154 inductive SPL4 (Fig. 4f). Since carbohydrate status regulates timing of the floral transition ${ }^{4}$, it is

155 interesting that T6P signaling also directly influences the re-organisation of the SAM during the

156 floral transition (Fig. 4g). Given the ubiquitous nature of carbohydrate signaling and the large-

157 scale change in sink-source relationships within the plants ${ }^{23}$, it will be interesting to determine if

158 this regulatory mechanism is widely present in the plant kingdom. 
Methods

161

\section{Materials and Methods}

162

163

164

165

166

167

168

169

170

171

172

173

174

175

176

177

178

179

\section{Plant material and growth conditions}

All Arabidopsis thaliana plants used for this study are of the Columbia accession (Col-0). The lines clv3-7 $7^{24}$, clv3-10 25 , 35S:amiRTPS1, CLV3:TPS1 ${ }^{4}$, and WUS:WUS:GFP 22 have been described previously as indicated. Plants were grown in controlled growth chambers at $22^{\circ} \mathrm{C}$ in long day (LD, 16h light/8h dark) or short day (SD, 8h dark/16h light) conditions with a light intensity of approximately $160 \mu \mathrm{mol} / \mathrm{m}^{-2} \mathrm{~s}^{-1}$ and a relative humidity of $60-65 \%$. Controlled induction of flowering was performed using a previously described shift protocol ${ }^{21}$.

Phenotypic analysis

Flowering time was defined as days to flowering (DTF), recorded when shoots were $0.5 \mathrm{~cm}$ high (bolting), and by scoring the total leaf number (TLN). The TLN is the sum of rosette leaves (RLN, rosette leaf number) and cauline leaves (CLN, cauline leaf number) per plant (Table S1). On average 20 plants of each genotype were analyzed in one experiment. Plants were grown in the conditions described above, and randomized every second day to avoid position effects.

\section{Generation of transgenic lines}

Generally, Col-0 plants were transformed by the floral dip method ${ }^{26}$. The presence of the transgene was confirmed by PCR and independent, single-insertion, homozygous T3 plants were selected and used for all further studies. Oligonucleotides used for cloning are provided in Table S2, those used for genotyping are given in Table S3. To generate the 35S:TPPJ and 
182 into the Gateway ${ }^{\circledR}$ entry vector pJLBlue reverse ${ }^{27}$ (pVW275 and pVW099), and recombined into

198 cloned into the pJF1031 binary vector ${ }^{30}$. Plants with homozygous deletions for SPL3, SPL4 or 
RNA extraction and cDNA synthesis

203

Total RNA form homogenized rosette or dissected SAM samples was isolated by a

204 phenol/chloroform extraction method using a modified TRIzol reagent with the following

205 composition: phenol 38\% (v/v), guanidine thiocyanate $0.8 \mathrm{M}$ ammonium thiocyanate $0.4 \mathrm{M}$,

206 sodium acetate $0.1 \mathrm{M} \mathrm{pH} 5.0$, glycerol 5\% (v/v), EDTA $5 \mathrm{mM} \mathrm{pH} \mathrm{8.0,} \mathrm{Na-lauroylsarcosine} \mathrm{0.5 \%}$

207 (v/v). This was then followed by a sodium acetate precipitation to improve RNA purity. cDNA

208 synthesis, preceded with removal of putative genomic DNA contamination with Dnase I Rnase-

209 free $\left(\right.$ Ambion $^{\mathrm{TM}}$ /Thermo Fisher Scientific, Waltham, Massachusetts, US), was carried out using a

210 SuperScript ${ }^{\mathrm{TM}}$ IV Reverse Transcriptase Kit (Thermo Fisher Scientific, Waltham, Massachusetts,

211 US) according to the manufacturer's instruction. For quantification of mature miR156 the

212 respective stem-loop primers (Table S4) were added to the cDNA synthesis reaction (1:1 with

213 oligo dT(18) primer) (Thermo Fisher Scientific, Waltham, Massachusetts, US) as previously

214 described $^{31}$.

$215 \quad$ RT-qPCR analysis

216 RT-qPCR analyses were performed with the ABI Prism $7900 \mathrm{HT}$ fast real time PCR system

217 (Applied Biosystems/Life Technologies, Darmstadt, Germany) using a Power SYBR ${ }^{\circledast}$ Green-PCR

218 Master Mix (Applied Biosystems/Life Technologies, Darmstadt, Germany) in a $10 \mu$ l reaction

219 volume for expression analyses and in a $5 \mu$ l volume for ChIP-PCRs. All oligos are listed in Table

220 S4 and Table S5 (RT-qPCR and ChIP-PCR respectively). All data were analyzed using the SDS 2.4

221 software (Applied Biosystems/Life Technologies, Darmstadt, Germany) applying the criteria

222 described by Czechowski and co-workers (2004). cDNA quality was determined with primers 
223 binding to the $3^{\prime}$ and 5' regions of GLYCERALDEHYDE-3-PHOSPHATE DEHYDROGENASE (GAPDH,

224 At1g13440), and samples with Ct 5'GAPDH/Ct 3'GAPDH values $>1$ were excluded from further

225 analyses. Expression values were calculated by the comparative Ct method using the reference

226 gene index (RGI) as previously described ${ }^{4}$ and POLYUBIQUITIN10 (UBQ10, At4g05320) for figure

$2273 \mathrm{C}$ and $\mathrm{S} 14^{32}$. The primer sequences for the reference and analyzed genes are given in the Table

228 S4.

229 RNA in situ hybridization

Wax embedding was performed using an automated processor and embedding system

231 (Leica EG1160, Solms, Germany). Sections of $8 \mu \mathrm{m}$ thickness were prepared using a rotary 232 microtome (Leica RM2265; Leica, Wetzlar, Germany).

Probes for RNA in situ hybridization were synthesized using the DIG RNA Labeling Kit

234 (Roche, Mannheim, Germany). For this the ORFs of the target genes were cloned into the 235 pGEM $^{\circledR}-\mathrm{T}$ Easy vector (Promega, Madison, Wisconsin, US) as a template according to

236 manufactures instructions (oligonucleotides and construct IDs listed in the Table S6) and the 237 sense and antisense RNA probes were synthetized using T7 and SP6 polymerases, respectively.

238 RNA in situ hybridizations were carried out as previously described ${ }^{4}$. The final sections were 239 imaged with an Olympus BX-61 microscope equipped with a DC View II digital camera (Olympus 240 Europa SE \& Co, Hamburg, Germany).

241 Chromatin immunoprecipitation (ChIP)

242 For ChIP-PCR, 100 apices per biological replicate (Col-0, clv3-7 and clv3-10) were collected 243 and snap frozen in liquid $\mathrm{N}_{2}$. Samples were fixed in $1 \%(\mathrm{v} / \mathrm{v})$ formaldehyde buffer $(10 \mathrm{mM}$ 
244 sodium phosphate buffer, $\mathrm{pH} 7 ; 50 \mathrm{mM} \mathrm{NaCl} ; 100 \mathrm{mM}$ sucrose) under vacuum. ChIP was

245 performed as previously described ${ }^{33}$ with modifications: Antibody incubation (anti-WUS; AS11

246 1759; Agrisera) was extended to $\mathrm{o} / \mathrm{n}$ at $4^{\circ} \mathrm{C}$ and incubation with Agarose beads (Protein A-

247 Agarose; sc-2001; Santa Cruz Biotechnology) to $6 \mathrm{~h}$ at $4^{\circ} \mathrm{C}$. Immunoprecipitated DNA was

248 analyzed by RT-qPCR assay. Data were analyzed using the SDS 2.4 software (Applied

249 Biosystems/Life Technologies, Darmstadt, Germany). Ct values of TPPJ promoter regions were

250 normalized to the $\mathrm{Ct}$ value of a region within the UBQ10 promoter. The $\%$ of enrichment was

251 calculated as relative to the input expression of the individual TPPJ promoter regions. The

252 following controls were performed: ChIP on 100 apices of Col-0, clv3-7 and clv3-10 omitting

253 addition of $A B$ and ChIP on $1.5 \mathrm{~g}$ of Col-0, clv3-7 and clv3-10 leaves (Fig. S. 10). Please note that

254 in contrast to the input samples there was no amplification in any of the other ChIP control

255 samples. Oligonucleotides are listed in the Table S5.

256 Transactivation assay

257 For the effector construct line, the WUS (At2g17950) coding sequence was PCR amplified

258 using the Phusion High-Fidelity DNA Polymerase (Thermo Fisher Scientific, Waltham,

259 Massachusetts, US). The resulting fragment was cloned into the PGEM $^{\circledR}$-Teasy vector (Promega,

260 Madison, Wisconsin, US) (pVW310), sub-cloned into the Gateway ${ }^{\circledR}$ entry vector pJLBlue

261 reverse $^{27}$ (pMML056) and recombined using the Gateway ${ }^{\circledR}$ LR clonase II Enzyme mix

262 (Invitrogen, Carlsbad, CA) into a Gateway ${ }^{\circledR}$ destination vector with 35S promoter (pMDC32)

263 (pMML058). For the reporter gene constructs, designated regions of the 5' TPPJ region were

264 amplified with specific primers (Table S2) using the Phusion High-Fidelity DNA Polymerase

265 (Thermo Fisher Scientific, Waltham, Massachusetts, US). The resulting Kpnl/Acyl fragment was 
266 sub-cloned into the Gateway ${ }^{\circledR}$ entry vector pMDC162 to obtain GUS gene fusions (Table S2).

267 The 35Somega:LUC-NOS plasmid containing the LUC gene driven by the 35S promoter was used

268 as an internal control. Col-0 protoplasts were isolated from leaves of 4-week-old plants and

269 transfected by a modified polyethylene glycol method ${ }^{34}$. The transfected cells were incubated

270 for $20 \mathrm{~h}$ at $22^{\circ} \mathrm{C}$ in the light $\left(100 \mu \mathrm{mol} / \mathrm{m}^{-2} \mathrm{~s}^{-1}\right)$, harvested by centrifugation at $100 \mathrm{~g}$ for $2 \mathrm{~min}$,

271 and then lysed ${ }^{35}$. Luciferase activity was measured with a luciferase assay kit (Promega,

272 Madison, Wisconsin, US) according to the manufacturer's instruction, and GUS activity was

273 determined according to a previously described protocol ${ }^{35}$.

274 Electrophoretic mobility shift assay (EMSA)

275 WUS coding sequence without STOP codon was PCR amplified using the Phusion DNA

276 polymerase (New England Biolabs). The resulting fragment was sub-cloned into the Gateway ${ }^{\circledR}$

277 entry vector pDONR207 (pMML059) and recombined into a Gateway ${ }^{\circledR}$ destination vector with

27835 s promoter (pDEST24) using the Gateway ${ }^{\circledR}$ LR clonase II Enzyme mix (Invitrogen, Carlsbad,

279 CA) resulting in pMML063. The plasmid was transformed into the Escherichia coli strain Rosetta

280 plys and the protein production was induced with $1 \mathrm{mM}$ isopropyl $\beta$-D-1-

281 thiogalactopyranoside at $30^{\circ} \mathrm{C}$ over-night. Bacterial cell lysis was performed by sonication (1x, 5

282 sec, 20\% power, 4 cycles, Sonoplus Hd 2070 Sonicator, Badelin, Berlin, Germany) preceded by

283 incubation on ice for $20 \mathrm{~min}$ in the freshly prepared protein extraction buffer $(20 \mathrm{mM} \mathrm{Na}$ -

284 phosphate buffer, $\quad \mathrm{pH} 7.4 ; \quad 0.5 \mathrm{M} \mathrm{NaCl} ; \quad 1 \mathrm{mM}$ phenylmethylsulfonyl fluoride; $1 \mathrm{mM}$

285 Ethylenediaminetetraacetic acid; 1 tablet of cOmplete ${ }^{\mathrm{TM}}$ Protease Inhibitor Cocktail (Merck,

286 Darmstadt, Grmany) per $10 \mathrm{ml}$ of the buffer). For the EMSA $2 \mu \mathrm{g}$ of protein (crude extract,

287 determined by Pierce ${ }^{\mathrm{TM}}$ BCA Protein Assay Kit, Thermo Fisher Scientific, Waltham, 
288 Massachusetts, US) was used. The EMSA was performed using 5'-IRDey-682-labeled, double-

289 stranded oligos of 50 bp spanning the putative WUS binding sites in the $5^{\prime}$ regulatory region of

290 TPPJ (Table S7). Binding reactions were carried out using the Odyssey ${ }^{\circledR}$ EMSA Kit (LI-COR $\left.{ }^{\circledR}\right)$

291 according to the manufacturer's instruction with a competitor to probe ratio of 1:200. Results

292 were visualized using an Oddysey Infrafed Imaging System (Li-Cor, Lincoln, NE).

293 Confocal microscopy

294 Apices from developmental series of Col-0 TCSn:GFP cytokinin reporter line lines were

295 excised using a diamond knife leaving a stalk of stem tissue. This stalk was used to fix the

296 sample for imaging in a droplet of $0.1 \%$ agar covered with Perfluorodecalin (F2 Chmicals,

297 Lancashire, UK) on a glass slide. A Leica SP8 confocal laser scanning system equipped with a

298 M6000B-CS microscopy stage, an Argon laser $(65 \mathrm{mV})$, and a 40x water immersion HCX APO

299 objective was used to image SAMs with following settings: Laser output power 20\%; GFP

300 Excitation (green): wavelength $488 \mathrm{~nm}$ / emission detection channel 3: 495 - $520 \mathrm{~nm}$, gain PMT

$301800 \mathrm{~V}$; plastid auto-fluorescence (blue) emission detection channel 4: 700 - $800 \mathrm{~nm}$, gain PMT

$302 \sim 500 \mathrm{~V}$; scan speed $600 \mathrm{~Hz}$ in xyz bi-directional scanning mode with a z-stack distance of

303 approx. $10 \mu \mathrm{m}$. Offset $=0$; pixel dimension: $1024 \times 1024$. To visualize the differences in GFP

304 presence middle sections of representative SAMs were extracted from z-stacks using the Fiji

305 software package ${ }^{36}$, version 2.0.0-rc-69/1.52 max-intensity Z-Projection function.

306 Statistical consideration

307 Statistical significance of flowering time and RT-qPCR data was analyzed both, by one-way 308 ANOVA (Analysis of Variance) with Tukey' Post Hoc HSD (Honestly Significant Difference) based 
309

310

311

312

313

314

315

on Tukey-Kramer correction (P value $<0.05$ ) and two-tailed Student's $t$-test. Significance of the data was marked with asterisks $\left({ }^{*}\right)$ based on the following criteria: $\mathrm{P} \leq 0,05:{ }^{*}, \mathrm{P} \leq 0,01: * *, \mathrm{P} \leq$ 0,001: ***. The presented statistical data are based on: flowering time experiments - min. 20 plants of each genotype; SAM area measurements - longitudinal middle section through min. 7 individual SAMs; WUS and CLV3 expression domain size and distance to the summit longitudinal middle section through min. 6 individual SAMs RT-qPCR - min. three biological replicates; Transactivation assay -6 biological replications; ChIP -3 biological replications.

\section{Data availability}

All data supporting the findings of this study are available in the main text or the Supplementary Information. All biological materials used in this study are available from the corresponding author on reasonable request. Source data are provided with this paper.

\section{References and Notes}

1 Kwiatkowska, D. Flowering and apical meristem growth dynamics. J Exp Bot 59, 187-201, doi:10.1093/jxb/erm290 (2008).

2 Srikanth, A. \& Schmid, M. Regulation of flowering time: all roads lead to Rome. Cellular and molecular life sciences : CMLS 68, 2013-2037, doi:10.1007/s00018-0110673-y (2011).

3 Ponnu, J. et al. The Trehalose 6-Phosphate Pathway Impacts Vegetative Phase Change in Arabidopsis thaliana. Plant J, doi:10.1111/tpj.14965 (2020).

4 Wahl, V. et al. Regulation of flowering by trehalose-6-phosphate signaling in Arabidopsis thaliana. Science (New York, N.Y.) 339, 704-707, doi:10.1126/science.1230406 (2013).

5 Olas, J. J. et al. Nitrate acts at the Arabidopsis thaliana shoot apical meristem to regulate flowering time. New Phytol 223, 814-827, doi:10.1111/nph.15812 (2019).

6 Fichtner, F. \& Lunn, J. E. The Role of Trehalose 6-Phosphate (Tre6P) in Plant Metabolism and Development. Annu Rev Plant Biol, doi:10.1146/annurev-arplant050718-095929 (2021). 
Pfeiffer, A., Wenzl, C. \& Lohmann, J. U. Beyond flexibility: controlling stem cells in an ever changing environment. Curr Opin Plant Biol 35, 117-123, doi:10.1016/j.pbi.2016.11.014 (2017).

8 Kinoshita, A. et al. Regulation of shoot meristem shape by photoperiodic signaling and phytohormones during floral induction of Arabidopsis. Elife 9, doi:10.7554/eLife.60661 (2020).

9 Brand, U., Grunewald, M., Hobe, M. \& Simon, R. Regulation of CLV3 expression by two homeobox genes in Arabidopsis. Plant Physiol 129, 565-575, doi:10.1104/pp.001867 (2002).

10 Leibfried, A. et al. WUSCHEL controls meristem function by direct regulation of cytokinin-inducible response regulators. Nature 438, 1172-1175, doi:10.1038/nature04270 (2005).

12 Pfeiffer, A. et al. Integration of light and metabolic signals for stem cell activation at

11 Snipes, S. A. et al. Cytokinin stabilizes WUSCHEL by acting on the protein domains required for nuclear enrichment and transcription. PLoS Genet 14, e1007351, doi:10.1371/journal.pgen.1007351 (2018). the shoot apical meristem. Elife 5, doi:10.7554/eLife.17023 (2016).

13 Zurcher, E. et al. A robust and sensitive synthetic sensor to monitor the transcriptional output of the cytokinin signaling network in planta. Plant Physiol 161, 1066-1075, doi:10.1104/pp.112.211763 (2013).

14 Brand, U., Fletcher, J. C., Hobe, M., Meyerowitz, E. M. \& Simon, R. Dependence of stem cell fate in Arabidopsis on a feedback loop regulated by CLV3 activity. Science (New York, N.Y.) 289, 617-619, doi:10.1126/science.289.5479.617 (2000).

15 Lohmann, J. U. et al. A molecular link between stem cell regulation and floral patterning in Arabidopsis. Cell 105, 793-803, doi:10.1016/s0092-8674(01)00384-1 (2001).

16 Sloan, J. et al. Structural basis for the complex DNA binding behavior of the plant stem cell regulator WUSCHEL. Nat Commun 11, doi:ARTN 2223

10.1038/s41467-020-16024-y (2020).

17 Yadav, R. K. et al. WUSCHEL protein movement mediates stem cell homeostasis in the Arabidopsis shoot apex. Genes Dev 25, 2025-2030, doi:10.1101/gad.17258511 (2011).

18 Hyun, Y., Richter, R. \& Coupland, G. Competence to Flower: Age-Controlled Sensitivity to Environmental Cues. Plant Physiol 173, 36-46, doi:10.1104/pp.16.01523 (2017).

19 Xie, Y. et al. FHY3 and FAR1 Integrate Light Signals with the miR156-SPL ModuleMediated Aging Pathway to Regulate Arabidopsis Flowering. Mol Plant 13, 483-498, doi:10.1016/j.molp.2020.01.013 (2020). Cell 37, 254-266, doi:10.1016/j.devcel.2016.04.001 (2016).

21 Schmid, M. et al. Dissection of floral induction pathways using global expression analysis. Development 130, 6001-6012, doi:10.1242/dev.00842 (2003). for noncell autonomous stem cell induction in Arabidopsis. Proc Natl Acad Sci U S A 
23 Fernie, A. R. et al. Synchronization of developmental, molecular and metabolic

24 Wisman, E., Cardon, G. H., Fransz, P. \& Saedler, H. The behaviour of the autonomous maize transposable element En/Spm in Arabidopsis thaliana allows efficient mutagenesis. Plant Mol Biol 37, 989-999, doi:10.1023/a:1006082009151 (1998).

25 Forner, J., Pfeiffer, A., Langenecker, T., Manavella, P. A. \& Lohmann, J. U. Germlinetransmitted genome editing in Arabidopsis thaliana Using TAL-effector-nucleases. PLoS One 10, e0121056, doi:10.1371/journal.pone.0121056 (2015).

26 Clough, S. J. \& Bent, A. F. Floral dip: a simplified method for Agrobacterium-mediated transformation of Arabidopsis thaliana. Plant J 16, 735-743, doi:10.1046/j.1365313x.1998.00343.x (1998).

27 Mathieu, J., Warthmann, N., Kuttner, F. \& Schmid, M. Export of FT protein from phloem companion cells is sufficient for floral induction in Arabidopsis. Curr Biol 17, 1055-1060, doi:10.1016/j.cub.2007.05.009 (2007).

28 Schwab, R. et al. Specific effects of microRNAs on the plant transcriptome. Dev Cell 8, 517-527, doi:10.1016/j.devcel.2005.01.018 (2005).

29 Wang, Z. P. et al. Egg cell-specific promoter-controlled CRISPR/Cas9 efficiently

30 Ruf, S. et al. High-efficiency generation of fertile transplastomic Arabidopsis plants.

31 Varkonyi-Gasic, E., Wu, R., Wood, M., Walton, E. F. \& Hellens, R. P. Protocol: a highly sensitive RT-PCR method for detection and quantification of microRNAs. Plant Methods 3, 12, doi:10.1186/1746-4811-3-12 (2007).

32 Czechowski, T., Bari, R. P., Stitt, M., Scheible, W. R. \& Udvardi, M. K. Real-time RT-PCR profiling of over 1400 Arabidopsis transcription factors: unprecedented sensitivity reveals novel root- and shoot-specific genes. Plant $J$ 38, 366-379, doi:10.1111/j.1365-313X.2004.02051.x (2004).

33 Kaufmann, K. et al. Chromatin immunoprecipitation (ChIP) of plant transcription factors followed by sequencing (ChIP-SEQ) or hybridization to whole genome arrays (ChIP-CHIP). Nat Protoc 5, 457-472, doi:10.1038/nprot.2009.244 (2010).

34 Wu, F. H. et al. Tape-Arabidopsis Sandwich - a simpler Arabidopsis protoplast isolation method. Plant Methods 5, 16, doi:10.1186/1746-4811-5-16 (2009).

35 Yoo, S. D., Cho, Y. H. \& Sheen, J. Arabidopsis mesophyll protoplasts: a versatile cell system for transient gene expression analysis. Nat Protoc 2, 1565-1572, doi:10.1038/nprot.2007.199 (2007).

36 Schindelin, J. et al. Fiji: an open-source platform for biological-image analysis. Nat Methods 9, 676-682, doi:10.1038/nmeth.2019 (2012). 
423 The authors wish to thank all members of the Wahl group for fruitful discussions, T. Seibert, M.

424 Schmid and P. Wigge for critical reading of the manuscript, D. Walter for input on statistical

425 analyses, K. Kontbay, M. Liang and J. Van Dingenen for their support with the $s p l$

426 CRISPR/Cas9 deletion lines, M. Molochko for sharing the 35Somega:LUC-NOS plasmid, J.

427 Forner for providing the $p J F 1031$ and $p J F 1033$ plasmids.

428 Funding: Work in the Wahl group was supported by the BMBF (SolaMI, 031B0191), the DFG

429 (SPP1530: WA3639/1-2, 2-1), and the Max Planck Society.

430 Authors contribution

431 VW conceived and designed the experiments and prepared the figures with contributions from

432 MML. VW and MML analyzed the data and wrote the manuscript. MML, KF, AK, and VW

433 performed all essential experiments, i.e. VW generated most of the lines used, except for

434 ML1:amiRTPPJ (KF), the spl CRISPR/Cas9 deletion lines (MML), and all crosses thereof

435 (MML). MML performed all transactivation assays, ChIP-PCRs, EMSAs and qPCR analyses.

436 MML, AK, KF, CA, and VW analyzed SAM sizes and scored flowering time. RNA in situ

437 hybridizations were performed by VW, AK, KF and MML. FK and VW took the confocal

438 images. All authors have read and commented on the text and figures within this manuscript.

\section{Competing interests}

440 Authors declare no competing interests.

441 Additional Information

442 Supplementary Information: Figures S1-S22, Tables S1-S7, References (1-3). 

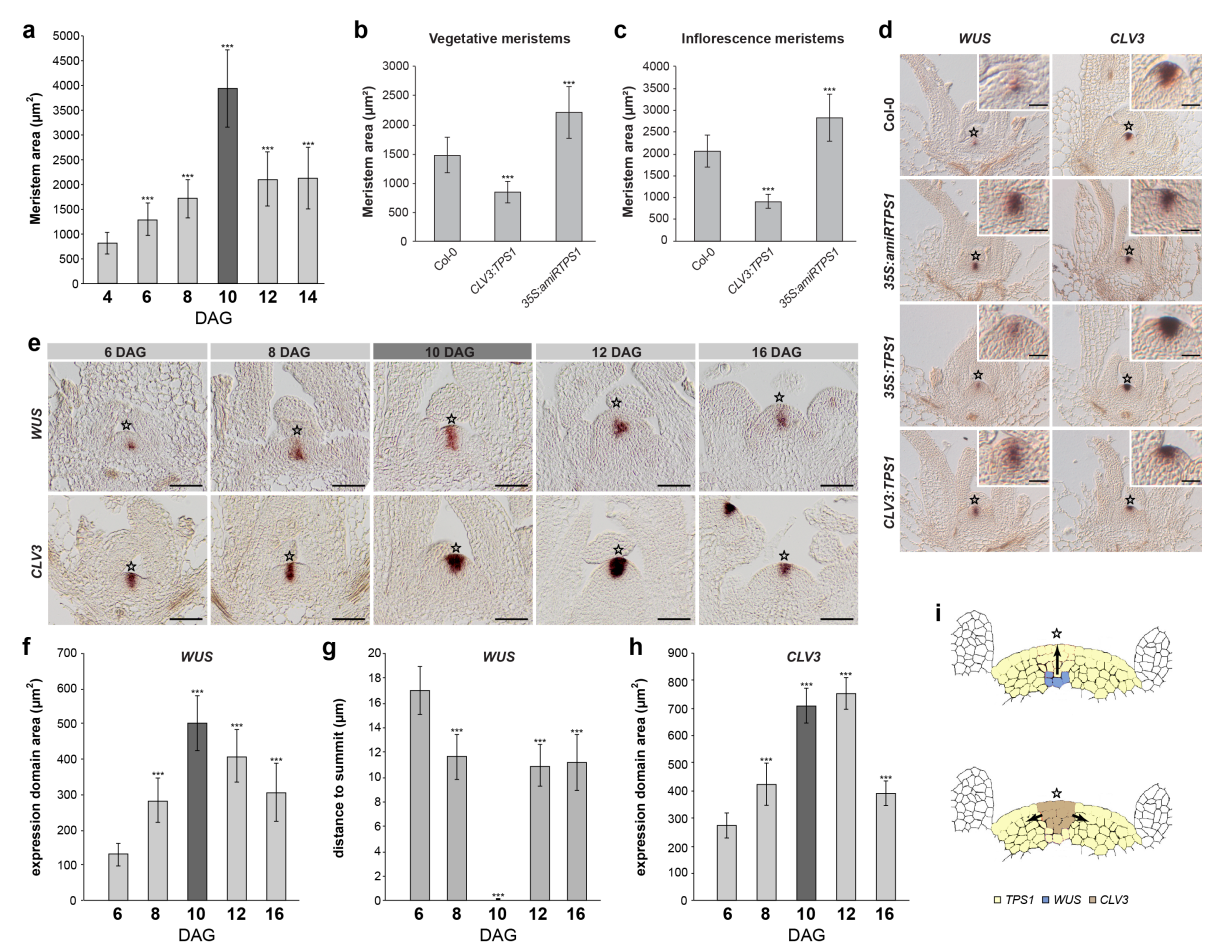

446

Fig. 1. The T6P pathway impacts SAM size during development. (a) SAM area throughout

447 development. (b) Vegetative and (c) inflorescence SAM size of CLV3:TPS1 and 35S:amiRTPS1

448 lines. (d) WUS and CLV3 expression by RNA in situ hybridisation on longitudinal sections

449 through Col-0, 35S:amiRTPS1, 35S:TPS1 and CLV3:TPS1 SAMs of LD-grown plants 8 days

450 after germination. (e) WUS and CLV3 expression by RNA in situ hybridisation in vegetative (6

451 and 8 DAG), transition (10 DAG, dark grey) and inflorescence SAMs (12 and 16 DAG) of LD-

452 grown Col-0 plants. (f) WUS expression domain sizes, (g) WUS expression domain distance to

453 SAM summit, and (h) CLV3 expression domain area, in vegetative, transition (dark grey) and

454 inflorescence SAMs of LD-grown wild-type plants. (i) Schematic picture of WUS, CLV3 and TPS1 expression dynamics with arrows indicating direction of increasing expression domain at

456 transition. Error bars denote s.d.; significance was calculated based on one-way Annova and a

457 Student's $t$-test, ${ }^{* * *} P<0.001$. 2 indicates SAM summit. Scale bars $25 \mu \mathrm{m}$. 


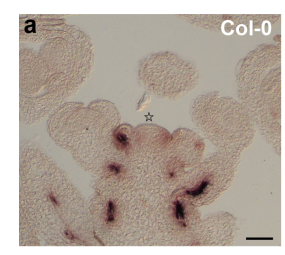

f

TPPJ

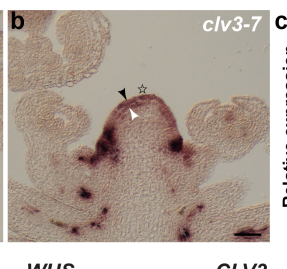

wus

CLV3

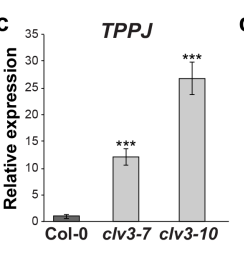

TPPJ

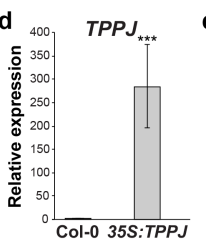

WUS

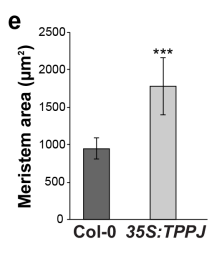

CLV3
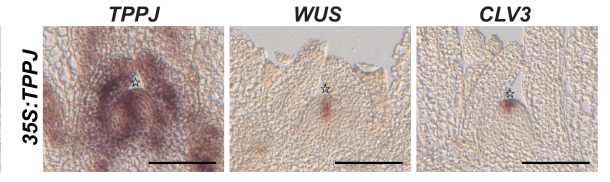

459 Fig. 2. WUS and $C L V 3$ expression in response to changes of TPPJ in the SAM. (a, b) TPPJ

460 expression by RNA in situ hybridisation on longitudinal sections through inflorescence SAMs of

461 Col-0 (a), and $\operatorname{clv3-7}(\mathbf{b})$, and by qRT-PCR in apices collected from $\operatorname{clv3-7,} \operatorname{clv3-10}$ (c), and

$46235 S: T P P J(\mathbf{d})$ plants. (e, f) SAM size of plants overexpressing TPPJ (e), expression of TPPJ,

$463 W U S, C L V 3$ by RNA in situ hybridization (f). Error bars denote s.d.; significance calculated by

464 one-way Anova and Student's $t$-test, $* * * P<0.001$. $46550 \mu \mathrm{m}$. 


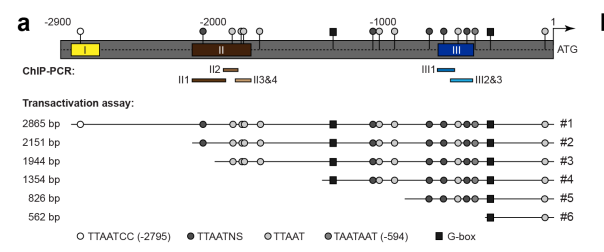

d
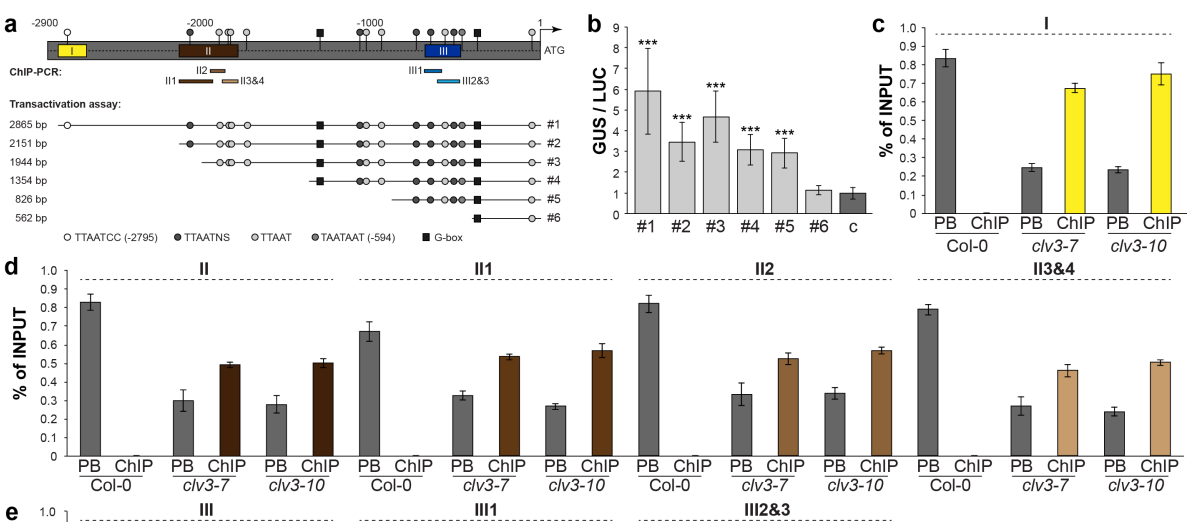

III
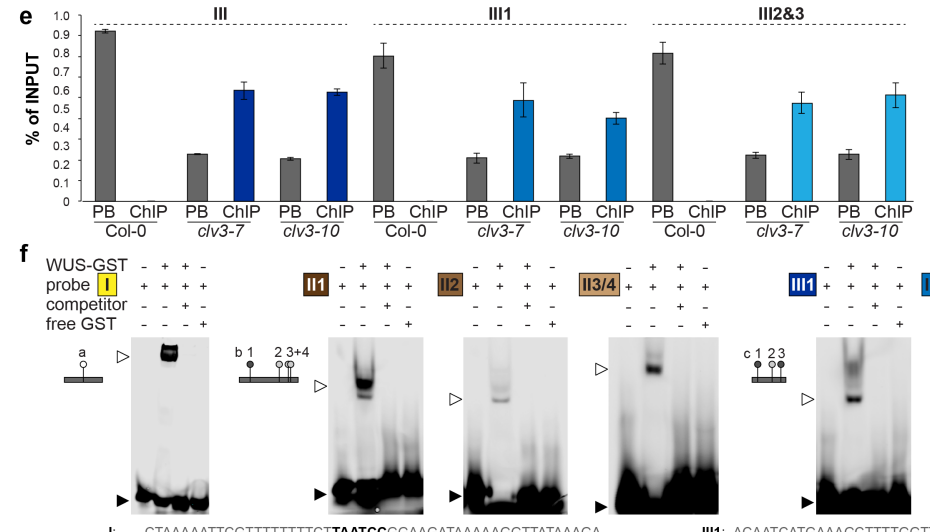

$[1314++++$

free GST

Fig. 3. WUS directly regulates TPPJ in the SAM. (a) Overview of TPPJ 5 ' regulatory region results shown in (c-e). Boxes marked with I, II, and III indicate 5' TPPJ regions with in total seven core WUS binding sites - I: $-2795--2789$ bp, II: $-2073--1830$ bp, and III: $-652--564$ bp. Sequence location and lengths used in (b) indicated with \#1-6. (b) Protoplast transactivation

472 assay showing activation of the GUS reporter when coupled to the regions indicated in (a),

473 relative to LUC activity. c indicates untransformed control. (c-e) Enrichment of (c) region I, (d)

474 region II, and (e) region III as indicated in (a) measured by ChIP-PCR relative to the input. PB 475 post binding fraction. (f) EMSA for WUS binding to the indicated regions (a, I-III). Error bars 476 denote s.d.; significance based on one way Anova and Student's $t$-test, $* * * P<0.001$. Scale bars 477 are $50 \mu \mathrm{m}$. 

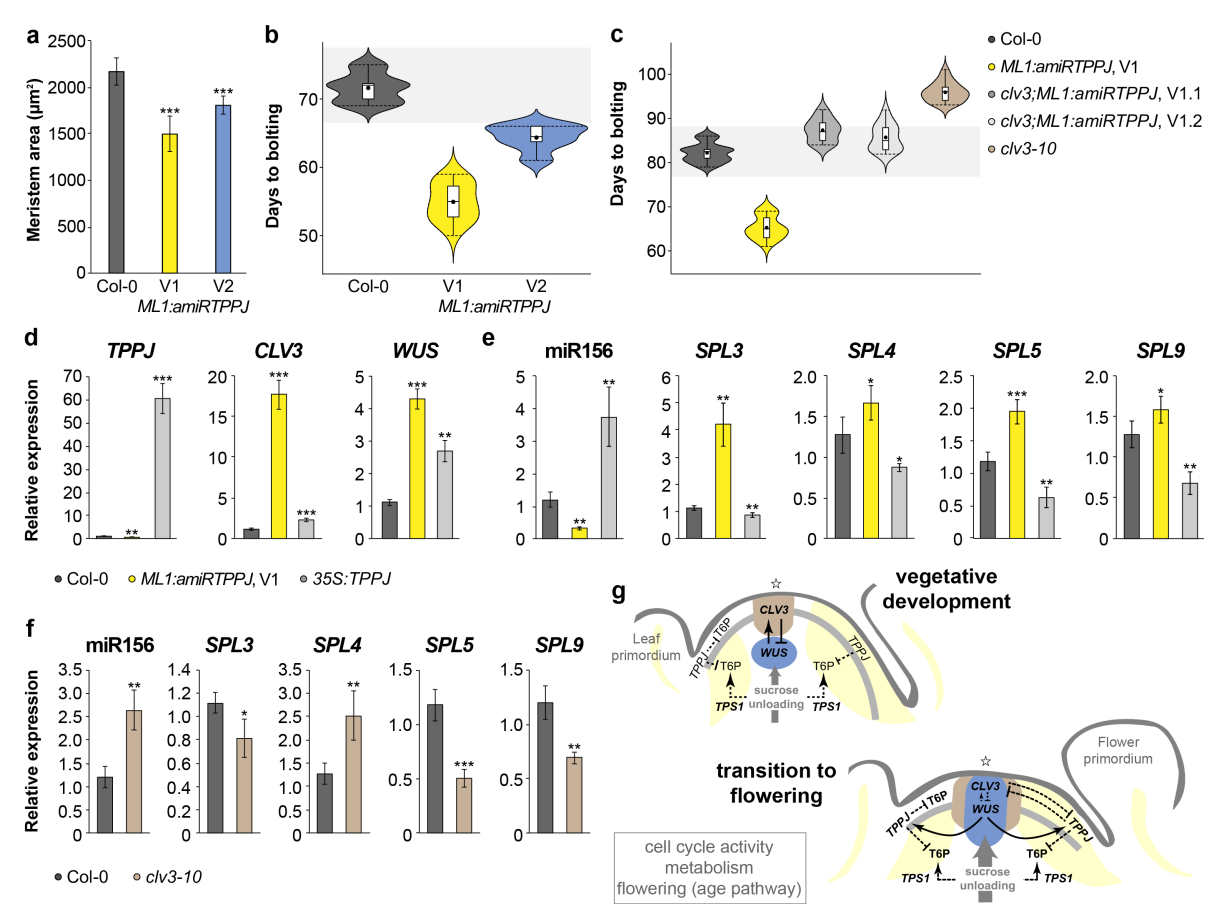

480

481

Fig. 4. The role of TPPJ in the outer SAM layer. (a) Meristem area of Col-0, ML1:amiRTPPJ

483 shown as days to bolting (a, c) relative to the wild type. V1 and V2 indicate two independent

484 versions of artificial microRNAs designed to target TPPJ transcript. (D, E) Relative expression

485 of SPL genes, mature miR156 (d), and TPPJ, CLV3, and WUS (E) in SD-grown ML1:amiRTPPJ

486 and 35S:TPPJ at 40 days after germination. (f) Relative expression of mature miR156 and SPL

487 genes in SD-grown clv3-10 at 40 days after germination. (g) Schematic model of vegetative and

488 transition SAM circuits. Error bars denote s.d.; significance calculated based on one way Anova

489 and Student's $t$ test; $* P<0.05, * * P<0.01, * * * P<0.001$. 次 indicates SAM summit. 